\title{
Retroperitoneal Fibrosis
}

National Cancer Institute

\section{Source}

National Cancer Institute. Retroperitoneal Fibrosis. NCI Thesaurus. Code C26876.

A rare, serious disorder characterized by extensive fibrosis in the retroperitoneum. In most cases its etiology is unknown. In some cases it is related to autoimmunity or metastatic cancer. It leads to the entrapment and obstruction of vital retroperitoneal structures including the ureters. 